\title{
MYXOMYCETES DO ESTADO DE RORAIMA, COM ESPECIAL REFERÊNCIA PARA A ESTAÇÃO ECOLÓGICA DE MARACÁ (AMAJARI - RR, BRASIL)
}

\author{
Laise de Holanda CAVALCANTI', Eneida Jucene dos SANTOS', Núbia \\ Abrantes GOMES'
}

RESUMO - Myxomycetes do Estado de Roraima, com especial referência para a Estação Ecológica de Maracá (Amajari - RR, Brasil). Este levantamento eleva para 28 o número de espécies de Myxomycetes encontradas para o estado de Roraima. Destas, 24 foram coletadas na Estação Ecológica de Maracá ( Lat. $3^{\circ} 15^{\prime}-3^{\circ} 35^{\prime} \mathrm{N}$, Long. $61^{\circ} 22^{\prime}-61^{\circ} 58^{\prime} \mathrm{WG}$ ). As seguintes familias e gêneros são registrados: Ceratiomyxaceae (Ceratiomyxa, $4 \mathrm{sp}$ ); Cribrariaceac (Cribraria, 1sp, Dictydium, 1sp); Didymiaceae (Didymium, 2 sp); Enteridiaceac (Lycogala, 1sp, Tubifera, $2 \mathrm{sp}$ ); Physaraceae (Craterium, 2sp, Fuligo, 1sp, Physarum, 2 sp); Stemonitaceae (Comatricha, 1sp, Stemonitis, 4 sp); Trichiaceae (Arcyria, $3 \mathrm{sp}$, Hemitrichia, $2 \mathrm{sp}$, Perichaena, 1sp, Trichia, 1sp). Uma familia, sete gêneros e onze espécies são novas referências para o estado; uma delas, Ceratiomyxa sphaerosperma Boedj. , é nova para o estado de Roraima e para a Região Norte. São apresentados comentários e a distribuição geográfica das espécies no Brasil.

Palavras-chave: Myxomycetes; florística; reservas ecológicas ; corologia ; Ceratiomyxa.

Myxomycetes From Roraima State, with Special Reference to the Maracá Ecological Reserve (Amajari - RR, Brazil)

ABSTRACT - This survey raises the number of registers referred to the State of Roraima to 28 species of Myxomycetes. Of these, 24 were collected in the Maraca Ecological Reserve (Lat. $3^{\circ} 15^{\prime}-3^{\circ} 35^{\prime} \mathrm{N}$, Long. $\left.61^{\circ} 22^{\prime}-61^{\circ} 58^{\prime} \mathrm{WG}\right)$. The following families and genera are registered: Ceratiomyxaceae (Ceratiomyxa, 4 sp); Cribrariaceae (Cribraria, 1sp, Dictydium, 1sp); Didymiaceae (Didymium, $2 \mathrm{sp}$ ); Enteridiaceae (Lycogala, 1 sp, Tubifera, 2 sp); Physaraceae (Craterium, 2 sp, Fuligo, 1sp, Physarum, 2 sp); Stemonitaceae (Comatricha, 1sp, Stemonitis, 4 sp);Trichiaceae (Arcyria, 3 sp, Hemitrichia, 2 sp, Perichaena, 1sp, Trichia, Isp). One family, seven genera and eleven species are new records for the State; one of them, Ceratiomyxa sphaerosperma Boedj., is new for Roraima State and Northern Brazil. Comments and geographical distribution of these species are presented.

Key-words: Myxomycetes; floristics; ecological reserves; chorology; Ceratiomyxa.

\section{INTRODUÇÃO}

Informações sobre a mixobiota de Roraima são escassas na literatura, não existindo ainda um trabalho especifico para este estado. Referências sobre a presença destes organismos podem ser encontradas em uns poucos trabalhos, como Crane \& Schoknecht (1982) que, ao descreverem Acrodontium myxomyceticola Crane \& Schok., informam que este
Hyphomycete se desenvolvera sobre esporângios de Stemonitis fusca Roth, coletado em 1977, a cerca de $219 \mathrm{~km}$ ao Norte de Boa Vista, capital do estado. Farr (1985) publicou uma relação de 26 espécies coletadas no antigo Território de Roraima e no estado do Amazonas, em excursões realizadas junto com pesquisadores nacionais e estrangeiros, participantes do Projeto Flora Amazônica; a listagem contém 11

\footnotetext{
I Departamento de Botânica, Universidade Federal de Pernambuco, Av. Prof. Moraes Rego s/n - Cidade Universitária, Recife - PE, Brasil. E-mail: 1hc@netpe.com.br.

2 Departamento de Biologia, Universidade Federal de Roraima, Campus do Paricarana, Boa Vista - RR.
} 
espécies coletadas em Roraima, distribuidas em oito gêneros, pertencentes às familias Ceratiomyxaceae, Cribrariaceae, Enteridiaceae, Trichiaceae, Physaraceae e Stemonitaceae; coleções representativas deste material estão depositadas no Herbário INPA (Manaus - AM), com duplicatas em herbários norte - americanos (NY, New York Botanical Garden; BPI, National Fungus Collections). Estas espécies não foram citadas para o estado de Roraima por Putzke (1996), que pretende apresentar uma lista atualizada das espécies referidas para o Brasil até a presente década, apesar de incluir o trabalho de Farr (1985) nas suas referências bibliográficas; no mesmo trabalho, o autor cita, erroneamente, a ocorrência de $S$. fusca no estado de Rondônia, baseando-se no trabalho de Crane \& Schoknecht (1982).

A Estação Ecológica de Maracá ( (Lat. $3^{\circ} 15^{\prime}-3^{\circ} 35^{\prime} \mathrm{N}$, Long. $61^{\circ} 22^{\prime}$ $61^{\circ} 58^{\prime} \mathrm{WG}$ ) foi criada em junho de 1981, abrangendo uma área de 101312 ha, pertencendo, atualmente, ao municipio de Amajari. A ilha onde se localiza a Estação, formada pela bifurcação do Rio Ururicoera, apresenta cobertura vegetal muito variada, compreendendo savanas, campinas, buritizais, capoeiras, matas de carrasco, floresta fluvial, floresta de terra firme, de várzea e de vazante, predominando a floresta tropical úmida e campos de gramíneas . A flora e fauna locais são muito diversificadas, abrigando espécies vegetais de grande valor econômico e animais ameaçados de extinção (Lewis \& Owen, 1989; Milliken \& Ratter, 1989). Em meados da década de 80 , B. Lowy e K. F. Rodrigues efetuaram coletas de Myxomycetes no lado leste da ilha, em áreas cobertas por florestas e savanas. Estas espécies estão relacionadas no presente trabalho, como também as citadas por Farr (1985), em conjunto com outras obtidas em coletas efetuadas na presente década pelas autoras na Estação Ecológica de Maracá. Algumas delas, como as pertencentes ao gênero Ceratiomyxa, têm distribuição restrita ou pouco conhecida no Brasil, e o registro em Roraima amplia o conhecimento sobre sua distribuição geográfica mundial , tornando, ao mesmo tempo, melhor conhecida a mixobiota brasileira.

\section{MATERIAL E MÉTODOS}

Amostras de Myxomycetes foram coletadas por N. A. Gomes e E. J. dos Santos entre 1990 -1992, na parte leste da ilha, nas proximidades da Casa Santa Rosa, ao longo da Trilha da Preguiça e da Trilha Central. As frutificações, encontradas sobre troncos mortos, folhas e frutos de vegetais vivos ou em decomposição, foram herborizadas e enviadas para o Departamento de Botânica da Universidade Federal de Pernambuco, para análise e identificação, efetuadas por L. H. Cavalcanti, segundo Martin \& Alexopoulos (1969) e Farr (1976), adotando-se o sistema de classificação de Martin et al. (1983). Material representativo encontra-se depositado no herbário UFP (Recife -PE).

Ao material analisado, acrescentouse a relação de espécies coletadas durante as expedições do Projeto Flora Amazônica, especialmente por K. P. Dumont, em diferentes pontos das rodovias Manaus - Boa Vista e Manaus - Caracarai, no trecho pertencente ao estado de 
Roraima, bem como o material obtido por B. Lowy e K.F. Rodrigues na década de 80 na ilha de Maracá, cujas exsicatas encontram-se depositadas no herbário INPA (Manaus-AM) .

A distribuição das espécies nos diferentes estados do Brasil baseou-se nos seguintes trabalhos: Bresadola (1896), Jahn (1902, 1904), Torrend (1915, 1916), Gottsberger (1968), Cavalcanti (1970, 1974, 1976, 1985), Pôrto (1982), Cavalcanti \& Araujo (1985), Cavalcanti \& Marinho (1985), Cavalcanti \& Oliveira (1985), Cavalcanti \& Silva (1985), Farr (1985), Rodrigues (1985), Santos et al. (1986), Capelari \& Maziero (1988), Cavalcanti \& Brito Jr. (1990), Rodrigues \& Guerrero (1990), Gottsberger et al. (1992), Cavalcanti \& Fortes (1994), Alves \& Cavalcanti (1996), Góes Neto (1994; 1996), Hochgesand \& Gottsberger (1996).

\section{RESULTADOS E DISCUSSÃO}

A subclasse Ceratiomyxomycetidae, com sua única família e gênero, está muito bem representada no estado de Roraima, especialmente na Estação Ecológica de Maracá, onde ocorrem as três espécies reconhecidas por Martin \& Alexopoulos (1969), das quais apenas $C$. fruticulosa tem ampla distribuição mundial (Tab. 1).

A presença de $C$. morchella e $C$. sphaerosperma no Brasil é mencionada por Martin \& Alexopoulos (1969) e Farr (1976), porém sem indicação da localidade, estado ou mesmo região em que foram assinaladas. Farr (1985) fez a primeira referência mais precisa da ocorrência de $C$. morchella para o Brasil, com base em coletas feitas no estado de Roraima, ao longo da rodovia Manaus - Boa Vista. Em 1990, N. A. Gomes coletou uma frutificação de $C$. sphaerosperma na Estação Ecológica de Maracá, sobre frutos em decomposição de Apeiba sp. No material deste gênero, coletado no mesmo local na década de 80 por B. Lowy e depositado no herbário INPA, encontrase uma amostra em cuja etiqueta consta a observação de ser uma possivel espécie nova, porém não foi possível identificá-la, dado o precário estado de conservação da exsicata, nem foi encontrada referência a respeito do novo taxon na literatura.

Da subclasse Myxogastromycetidae se tem registro de três ordens para o estado de Roraima e ilha de Maracá : Liceales, com as familias Enteridiaceae (Tubifera, Lycogala) e Cribrariaceae (Cribraria, Dictydium); Trichiales, com a familia Trichiaceae (Arcyria, Hemitrichia, Perichaena, Trichia) e Physarales, com as familias Physaraceae (Craterium, Fuligo, Physarum) e Didymiaceae (Didymium).

Boa parte das espécies da subclasse Myxogastromycetidae até agora coletadas em Roraima tem ampla distribuição mundial, sendo conhecidas para diferentes regiões do Brasil ; todavia, várias delas, assinaladas com um asterisco na Tabela 1 , não haviam sido referidas para o estado. O registro de L. epidendrum e $T$. microsperma complementa as informações de Cavalcanti \& Brito Jr. (1990) sobre a área de distribuição das Enteridiaceae no Brasil.

A subclasse Stemonitomycetidae, já assinalada no estado, também ocorre na Estação Ecológica de Maracá, representada por espécies dos gêneros Comatricha e 
Tabela 1. Myxomycetes ocorrentes no Estado de Roraima e sua distribuição no Brasil.

Convençōes: * = Nova referência para Roraima. (1)= K.P. Dumont : a - ao longo da rodovia Manaus - BoaVista (BR 174); b - $219 \mathrm{~km} \mathrm{~N}$ de Boa Vista, BR 174; c - Rodovia Manaus - Caracaraí, Acampamento do $6^{\circ}$. BEC Jundiá; d - BR 174, 204km N de Boa Vista. (2) = N.A. Gomes, ilha de Maracá. (3) = E. J. Santos, ilha de Maracá. $(4)=$ B. Lowy, ilha de Maracá. (5) = B. Lowy \& K.F. Rodrigues, ilha de Maracá.

Nomes dos estados, em ordem alfabética, abreviados segundo a Fundaçăo Instituto Brasileiro de Geografia e Estatística: Acre (AC); Alagoas (AL); Amazonas (AM); Bahia (BA); Ceará (CE); Minas Gerais (MG); Pará (PA): Paraiba (PB); Pernambuco (PE); Paraná (PR); Rio de Janeiro (RJ); Rondônia (RO); Roraima (RR); Rio Grande do Sul (RS); Santa Catarina (SC); São Paulo (SP).

\begin{tabular}{|c|c|c|}
\hline TAXON & COLETOR & DISTRIBUIÇÃO NO BRASIL \\
\hline \multicolumn{3}{|l|}{ Arcyria } \\
\hline A. cinerea (Bull.) Pers. & $(1 \mathrm{a}) ;(1 \mathrm{~b}):(1 \mathrm{c}) ;(2)$ & $\begin{array}{l}\text { AC,AL,AM,BA,CE,PA,PB, } \\
\text { PE,PR,RJ,RR, RS,SC,SP. }\end{array}$ \\
\hline A. denudata (L.) Wetts. & (1 a ); (1c); (2); (5) & $\begin{array}{l}\text { AM,BA,PB,PE,PR,RJ,RO, } \\
\text { RR,RS,SC,SP, }\end{array}$ \\
\hline $\begin{array}{l}\text { Arcyria sp. } \\
\text { Ceratiomyxa }\end{array}$ & (5) & RR \\
\hline C. fruticulosa (Mull.) Macbr. & $(1 \mathrm{a}) ;(1 \mathrm{c}) ;(2) ;(4)$ & $\begin{array}{l}\text { AL,AM,AP,BA,CE,PA,PB,PE } \\
\text { RN,RR,RS, SC, SP. }\end{array}$ \\
\hline C. morchella Welden & $(1 \mathrm{a}):(4)$ & AM,RR. \\
\hline C. sphaerosperma* Boedjin & & RR \\
\hline $\begin{array}{l}\text { Ceratiomyxa sp. nov.? } \\
\text { Comatricha* }\end{array}$ & (4) & RR \\
\hline $\begin{array}{l}\text { C. typhoides* (Bull.) Rost. } \\
\text { Craterium }^{*}\end{array}$ & (2) & $A M, C E, P B, P E, P R, R R, R S, S P$. \\
\hline C. aureum* (Schw.) Rab. & (3) & AM,BA,PB,PE,PR,RR,RS,SC,SP. \\
\hline $\begin{array}{l}\text { C. leucocephalum " (Pers.) Ditm, } \\
\text { Cribraria* }\end{array}$ & (3) & $\mathrm{AM}, \mathrm{BA}, \mathrm{PB}, \mathrm{PE}, \mathrm{RR}, \mathrm{SP}, \mathrm{SC}, \mathrm{RS}$ \\
\hline $\begin{array}{l}\text { C. microcarpa* (Schrd.) Pers. } \\
\text { Dictydium }\end{array}$ & (3) & PB,PE,PR,RR,SP. \\
\hline $\begin{array}{l}\text { D. cancellatum (Batsch)Macbr. } \\
\text { Didymium* }\end{array}$ & $(1 \mathrm{c})$ & $A M, B A, C E, P B, P E, P R, R J, R R, S P$ \\
\hline D. clavus*(Alb. \& Schw.) Rab. & (3) & BA,PE,PR,RR,RS,SC,SP. \\
\hline \multicolumn{3}{|l|}{ Fuligo } \\
\hline F. septica (L.) Wigg. & (4) & $\begin{array}{l}\text { AL,AM,BA,CE,MG,PE,PR, } \\
\text { RR,RS,SC,SP. }\end{array}$ \\
\hline \multicolumn{3}{|l|}{ Hemitrichia } \\
\hline H. calyculata (Speg.) Farr & (1 a): (1c); (2) & $\begin{array}{l}\text { AM,BA,CE,PB,PE,PR,RJ,RR, } \\
\text { RS,SC,SP. }\end{array}$ \\
\hline $\begin{array}{l}\text { H. serpula* (Scop.) Rost. } \\
\text { Lycogala }\end{array}$ & (2) & $A M, B A, P E, P R, R J, R R, R S, S C, S P$. \\
\hline L. epidendrum (L.) Fries & (1d) & $\begin{array}{l}\text { AL,AM,BA,PB,PE,PR,RJ,RR, } \\
\text { RS,SC,SP }\end{array}$ \\
\hline \multicolumn{3}{|l|}{ Perichaena } \\
\hline $\begin{array}{l}\text { Perichaena sp. } \\
\text { Physarum }\end{array}$ & (4) & $\mathrm{RR}$ \\
\hline P. nucleatum Rex & $(1 \mathrm{a}) ;(2)$ & $A M, C E, P E, P R, R R, S P$. \\
\hline $\begin{array}{l}\text { P. pezizoideum (Jung.) P.\&Lag. } \\
\text { Stemonitis }\end{array}$ & $(2) ;(4)$ & $\mathrm{BA}, \mathrm{PE}, \mathrm{RR}, \mathrm{RS}, \mathrm{SC}$ \\
\hline S. axifera (Bull.) Macbr. & (1b) & AM,BA,MG,PR,RJ,RR,SC,SP \\
\hline S. flavogenita* Jahn & & PB,PE,RR,SP. \\
\hline S. fusca Roth & (1 a); (2) & $\begin{array}{l}\text { AM,BA,PA,PB,PE,PR,RJ,RR, } \\
\text { RS,SC,SP. }\end{array}$ \\
\hline $\begin{array}{l}\text { S. smithii* Macbr. } \\
\text { Trichia* }\end{array}$ & (2) & MG,PE,PR,RJ,RR,SC, SP. \\
\hline $\begin{array}{l}\text { T. favoginea* (Batsch) Pers. } \\
\text { Tubifera }\end{array}$ & (2) & BA,PE,RJ,RR,RS,SC,SP. \\
\hline $\begin{array}{l}\text { Tubifera microsperma } \\
\text { (Berk. \& Curt.) Martin }\end{array}$ & $(1 \mathrm{a}):(1 \mathrm{c})$ & $\begin{array}{l}\text { AM,BA,PA,PB,PE,PR,RR,RS, } \\
\text { SC,SP. }\end{array}$ \\
\hline Tubifera sp & (4) & RR \\
\hline
\end{tabular}


Stemonitis, da familia Stemonitaceae (Tab. 1). São todas espécies de ampla distribuição mundial, porém $S$. flavogenita ainda não tinha sido encontrada na Região Norte do Brasil.

A família Didymiaceae e os gêneros Comatricha, Craterium, Cribraria, Didymium, Fuligo, Perichaena e Trichia estão sendo citados pela primeira vez como presentes na Estação Ecológica de Maracá e no estado de Roraima.

Na Tabela 1 estão relacionados os gêneros e espécies encontrados no estado de Roraima até o momento, com respectivos coletores, acompanhados da distribuição conhecida para cada espécie no Brasil. Apenas quatro espécies ( $T$. microsperma, $S$. axifera, D. cancellatum, L. epidendrum ), dentre as referidas para o estado, não têm registros para a Estação Ecológica de Maracá.

\section{Bibliografia Citada}

Alves, M. H.; Cavalcanti, L. de H. 1996. Myxomycetes em palmeiras (Arecaceae). Acta Botanica Brasilica, 10 (1): 1-7.

Bresadola, J. 1896. Fungi brasiliensis lecti a cl. Dr. Alfred Möller. Hedwigia, 35(5):276-302.

Capelari, M.; Maziero, R. 1988. Fungos macroscópicos do Estado de Rondônia, região dos rios Jaru e Ji-Paraná. Hoenea, I5: 28-36.

Cavalcanti, L. de H. 1970. Coleção de Mixomicetos do Museu Paraense Emilio Goeldi. Boletim do Museu Paraense Emilio Goeldi, Sèrie Botânica, 35: 1-5.

1974. Mixomicetos corticicolas do cerrado de Emas (Pirassununga - Estado de São Paulo). Dissertação de Mestrado, Instituto de Biociências, Universidade de São Paulo, São Paulo, 114p.

.... 1976. Mixomicetos novos para Pernambuco. II. Universidade Federal de Pernambuco, Ciências Biológicas, Recife, Série Botânica, 4 (4): 1-19.
.... 1985. Mixomicetos do cerrado III. Revisão de literatura. In: ANON (ed.). Anais do $33^{\circ}$ Congresso Nacional de Botänica. Sociedade Botânica do Brasil, EMBRAPA, Brasilia, p. 173-179.

Cavalcanti, L. de H. ; Araújo, V. C. F. 1985. Myxomycetes da Paraiba II - Liceales. In: ANON (ed.) Anais da $8^{\circ}$ Reunião Nordestina de Botânica. Sociedade Botânica do Brasil, Seccional Pemambuco, Recife, p. 193 - 198.

Cavalcanti, L. de H.; Brito Júnior, S. C. 1990. Enteridiaceae do Brasil. Biologica Brasilica, 2 (2): 241-260.

Cavalcanti, L. de H.; Fortes, S. T. 1994. Myxomycetes de Florianópolis, Santa Catarina. Acta Botanica Brasilica, 8 (1): 65-75.

Cavalcanti, L. de H. ; Marinho, M. das G. V. 1985. Myxomycetes da Paraiba I - Trichiales. In: ANON (ed.). Anais da $8^{\circ}$ Reunião Nordestina de Botânica. Sociedade Botânica do Brasil, Seccional Pernambuco, Recife, p. 185 -191.

Cavalcanti, L. de H. ; Oliveira, I. C. 1985. Myxomycetes da Paraiba IV - Stemonitales. In: ANON (ed.). Anais da $8^{\circ}$ Reunião Nordestina de Botânica. Sociedade Botânica do Brasil, Seccional Pernambuco, Recife, p. 297-214.

Cavalcanti, L. de H.; Silva, J. V. B. 1985. Myxomycetes da Paraiba III - Physarales. In: ANON (ed.). Anais da $8^{\circ}$ Reunião Nordestina de Botânica. Sociedade Botânica do Brasil, Seccional Pernambuco, Recife, p.199-205.

Crane, J. L. ; Schoknecht, J. D. 1982. A new species of Acrodontium from Brazil. Transactions of the Britsh Mycological Society, 79 (2) : 345-347.

Farr, M. L. 1976. Myxomycetes. Flora Neotropica . New York, New York Botanical Garden (Monograph n- 16$)$. 304p.

1985. Notes on Myxomycetes IV. Species collected in Brazil and Japan. Nova Hedwigia, 41: 167-175.

Góes Neto, A. 1994. Diagnóstico da biodiversidade de macromicetos do Estado da Bahia: evolução histórica e situação atual. Monografia de Bacharelado, Curso de Ciências Biológicas. Universidade Federal da Bahia, Salvador, Bahia, 75p.

.... 1996. Biodiversidade de mixomicetos e fungos 
macroscópicos da Reserva Biológica de Una e áreas adjacentes (Bahia, Brasil). Sitientibus, 15: 91- 108.

Gottsberger, G. 1968. Myxomyceten aus Bahia und Goiás. Nova Hedwigia, 15: 361-368.

Gottsberger, G. ; Schimidt, 1. ; Meijer,A. A. R. 1992. Macromycetes from the State of Paraná, Brazil. 2. Myxomycetes. Arquivos de Biologia e Tecnologia, 35 (4): 631- 633.

Hochgesand, E;; Gottsberger, G. 1996. Myxomycetes from State of São Paulo, Brazil. Boletim do Instituto de Botânica, 10: 1-46.

Jahn, E. 1902. Myxomycetenstudien. 2. Arten aus Blumenau (Brasilien). Berichte der Deutschen botanischen Gesellschafi, 20: 268-280.

1904. Myxomyceten aus Amazonas. Hedwigia , 43 (4/5): 300-305.

Lewis, G. P. ; Owen, P. E. 1989. Legumes of the Ilha de Maracá. Kew, Royal Botanic Gardens. 95p.

Martin, G. W. ; Alexopoulos, C. J. 1969. The Myxomyceles. lowa City, University of lowa Press. $561 \mathrm{p}$.

Martin, G. W.; Alexopoulos, C. J.; Farr, M. L. 1983. The Genera of Myxomycetes. Iowa City, University of lowa Press. 570p.

Milliken, W.; Ratter, J. A. 1989. Vegetation of the Ilha de Maracá: first report of the vegetation survey of the Maraca rain forest Project (INPA / RGS / SEMA). Edinburg, Royal Botanic Garden. 277p.
Pôrto, K.C. 1982. Myxomycetes da Mata de Dois Irmãos (Recife-Pernambuco). Dissertação de Mestrado, Departamento de Biologia. Universidade Federal Rural de Pernambuco, Recife, Pernambuco, 367p.

Putzke, J. 1996. Myxomycetes do Brasil. Caderno de Pesquisa, Série Botânica, 8 (1/2): 3-85.

Rodrigues, K. 1985. Contribuição ao estudo dos Mixomicetos do Estado do Rio de Janeiro. Rodriguesia , 37 (62): 46-47.

Rodrigues, C. L. M. ; Guerrero, R. T. 1990. Myxomycetes do Morro Santana, Porto Alegre, Rio Grande do Sul. Boletim do Instituto de Biociências, 45: 1-102.

Santos, E. J.; Cavalcanti, L. de H.; Albuquerque, W. C. 1986. Myxomycetes de Alagoas. In: ANON (ed.). Anais do $33^{\circ}$ Congresso Nacional de Botânica. Sociedade Botânica do Brasil, Ouro Preto, p. 499 -504.

Torrend, C. 1915. Les Myxomycètes du Brasil, connus jusquici. Broteria, Série Botânica, 13: $72-88$.

1916. Os mixomicetas dos arredores da Bahia. In: ANON (ed.). Anais do $5^{\circ}$ Congresso Brasileiro de Geografia. Sociedade Brasileira de Geografia, Salvador, p. 484-492. 